\title{
Research and Application of One-key Sequence Control Technology for Substations
}

\author{
Ge Li-Qing, Wang Jian-Feng, Teng Jing-Yu, Yang Ming \\ NR Electric Co., Ltd, NanJing, 211102 China
}

\begin{abstract}
The substations are important parts of modern electrical grids. In this sense, it is necessary to enhance the management efficiency and robustness of the substations. The one-key sequence control technology system could simultaneously control several subsystems and make use of their functions to automatically operate the substations. In this study, three subsystems, i.e., monitoring subsystems, error analysis subsystem, and decision support subsystem are designed in the one-key sequence control technology system. All the background information from the substations is monitored, checked, detected to find the potential threats. The decision support subsystem provides suggestive ways to handle these problems. Therefore, through the reasonable use of the one-key sequence control technology, the overall effectiveness and efficiency of the substations can be enhanced. With the development of artificial intelligence technologies, the one-key sequence control technology system can be further improved with more powerful functions.
\end{abstract}

\section{Introduction}

The safety of modern electric grids is closely related to people's daily life. To improve the efficiency of the operation of electric systems, many intelligent substations have been built these years. The systems become more and more complex, which needs more human labors. Under this situation, the one-key sequence control technology provides a suitable way for the management of the large number of substations. Based on the one-key sequence control technology, the system could intelligently sense the states of the substations and adaptively make the responses. In the previous works, some researchers made efforts to enhance the automation level of the substations including automatic fault diagnosis, visualization, anomaly detection, etc. However, they only focused on single aspects and lack considerations on the whole system. Nowadays, the onekey control technologies have been a hot topic in the field of intelligent applications, which can be used in the management of substations. The one-key sequence control technology takes the systems in the substations as a whole and considers all the relevant operations and managements. Through the successful use of the one-key sequence control technology, the labor strength can be greatly relieved and the precision, effectiveness, and efficiency of the whole system can be improved.

In this paper, we research on the one-key sequence control technology of the substations. First, the components and overall design of the one-key sequence control technology are discussed. From the aspect of components, the one-key sequence control technology system should include the monitoring subsystem, error analysis subsystem, decision support subsystems, etc. These subsystems work based on the background information from the substations and detect the anomaly in the data flows. When an error occurs, the one-key sequence control technology system could quickly locate it and find a suitable way to handle it, which guides the workers to make correct decisions. Second, the operation procedure of the one-key sequence control technology is explained, which sequentially unitizes different subsystems to perform different jobs. The one-key sequence control technology employs all the subsystems as a whole so the effectiveness can be fully played. Finally, some newly popular new technologies in the field of artificial intelligence are discussed, which have potential in the one-key sequence control technology substations.

\section{Combination of One-key Sequence Control Technology}

\subsection{Monitoring subsystem}

The monitoring subsystem monitors the background information in the substations. As there is a large amount of information, the monitoring subsystem classify it into several categories. For example, the information can be divided into ones related to hardware, software, operating system, networks, etc. In addition, this subsystem also provides interfaces for the workers so they can observe the system states intuitively. The important information can be highlighted to help the 
workers catch the key conditions of the substations. The monitoring subsystem operates unstoppably to sense all the states of the substations and provide information for other subsystems.

\subsection{Error analysis subsystem}

Based on the labeled information from the monitoring subsystem, the error analysis subsystem detects the anomaly from the data flows in each category and make analysis. According to the historical data from the substations, the abnormal data flows can be detected using anomaly detection algorithms. Furthermore, the detected anomaly is identified with a more concrete type and fed back to the works. The works can find the problems and the related component. Then, they can take corresponding measures to handle the problems

\subsection{Decision support subsystem}

The decision support subsystem makes more detailed analysis on the reported anomaly and errors in the error analysis subsystem. The detected anomaly is analysed using some artificial intelligence technologies and determine its type. Based on the historical data and some expert system, the decision support subsystem could provide some reasonable advices for handling the present problems. These advices will be reported to the workers to support them make decisions. Also, the system could record these error events to further learn a more robust supporter.

\section{Operation Procedure of One-key Sequence Control Technology}

The one-key sequence control technology system operates based on the functional subsystems. All the subsystems are considered in a unified framework to fully make their effects. Fig. 1 shows the general procedure of the operation of the one-key sequence control technology system, which could be summarized as following steps:

Step 1: The monitoring subsystem checks the data flows from the substations and classify them as several categories.

Step 2: The error analysis subsystem detects the anomaly from the dataflows and locate them. The results are reported to the decision support subsystem.

Step 3: The decision support subsystem identifies the reported error from Step 2 and provides suggestive ways to handle the problem.

Step 4: The whole system recorded the whole event to further strengthen the capability.

Through the iteration and repetitive learning, the onekey sequence control technology system will be more robust and effective. All the subsystems operate automatically to monitor the data flows, detect the potential threats and locate the true errors. So, the onekey sequence control technology system could help the workers quickly solve the problems occurred in the substations.

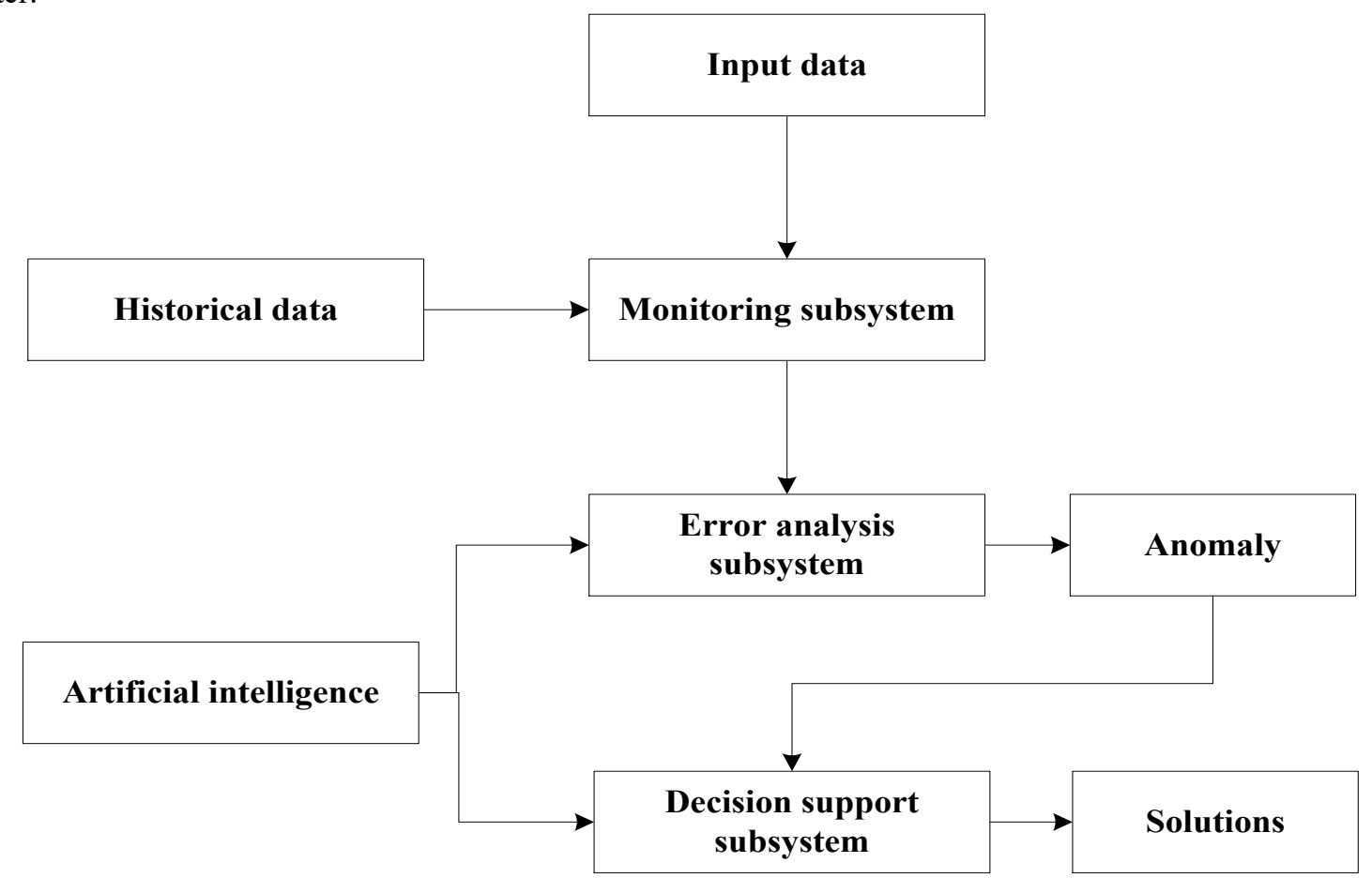

Fig. 1 General procedure of the one-key sequence control technology system in the substations.

\section{Conclusion}

This study researches on the one-key sequence control technology of the substations. In the one-key sequence control technology system, the monitoring subsystem, error analysis subsystem, and decision support system work cooperatively to find the potential problems occurred in the substations while providing reasonable ways to handle them. With the help of the one-key 
sequence control technology system, the workers could greatly enhance the working efficiency and quickly solve the problems. In the future, more artificial intelligence technologies could be applied to the one-key sequence control technology system such as deep learning, big data, etc. With better learning capabilities, the system could be more robust and effective.

\section{Acknowledgement}

Funding: This work was supported by Technology Funding Project of State Grid Corporation of China (Grant No. 52153218000G).

\section{References}

1. Borlea I, Buta A and Dusa et al. 2005 DIASE-Expert system fault diagnosis for Timisoara $200 \mathrm{kV}$ Substation in Proc. EUROCON pp 221-224.

2. Wang Y and Pan Z 2011 Study on test technology of smart substation secondary system in Proc. International Conference on Consumer Electronics, Communications and Networks pp 1-4.

3. Kezunovic M 2009 Substation fault analysis requirement in Proc. IEEE Power \& Energy Society General Meeting pp 1-2.784-787

4. Lian J, Li H and Song K 2011 The analysis and corrective measures of common problems of $10 \mathrm{kV}$ capacitor bank in substation in Proc International Conference on Advanced Power System Automation and Protection pp 1750-1752

5. Gogan P L and Wyckoff G D 2012 Design and construction of sustainable substations in Proc. PES T\&D pp 1-4.

6. Li Q, Zhou Z and Du D, et al. 2013 A novel substation area backup protection for smart substation in Proc. IEEE PES Asia-Pacific Power and Energy Engineering Conference pp 1-4.

7. Wang Z, Jin $\mathrm{N}$ and Zhang J, et al. 2014 Fault information and diagnosis modelling of on-line communication monitoring system for digital substation in Proc. IEEE PES General Meeting | Conference \& Exposition pp 1-5.

8. Liu Y, Gao H and Gao W, et al. 2017 Development of a substation-area backup protective relay for smart substation IEEE Transactions on Smart Grid vol 8 no 6 pp 2544-2553.
9. Zhang H, Dou R and Zhi M, et al. 2016 The technology of interlocking logic visualization for smart substation in Proc. China International Conference on Electricity Distribution pp 1-4.

10. Dehbonei H 2016 Earthing-Substation fire and station transformer customers supply transfer voltage issue in Proc. Down to Earth Conference pp 1-7.

11. Vardhan H and Ramlachan R 2018 Deploying digital substations: Experience with a digital substation pilot in north America in Proc. Annual Conference for Protective Relay Engineers pp 1-9.

12. Li Z and Ma Q 2018 Discussion on optimization measures of relay protection technology in intelligent substation in Proc. IEEE Advanced Information Management, Communicates, Electronic and Automation Control Conference pp 1593-1596.

13. Topolsky D V, ToPolskaya I G and Topolsky N D 2018 Development of an intelligent measuring system for digital substations in Proc. pp International Multi-Conference on Industrial Engineering and Modern Technologies 1-4.

14. Nassu B T, Lourival L and Bruno M et al. 2018 Image-based state recognition for disconnect switches in electric power distribution substations in Proc. SIBGRAPI Conference on Graphics, Patterns and Images pp 432-439.

15. Wang H, Li J and Zhou Y, et al. 2019 Research on the technology of indoor and outdoor integration robot inspection in substation in Proc. IEEE 3rd Information Technology, Networking, Electronic and Automation Control Conference pp 2366-2369.

16. Nichani A M and Swarup S 2018 Modelling and simulation of digital substation automation for intersubstation line protection in Proc. National Power Systems Conference pp 1-6.

17. Huang R, Zhang X and Yang H, et al. 2017 One Key Reset System for Substation Robot Based on Multi-sensor Fusion International Conference on Mechanical, Control and Computer Engineering pp $1-4$.

18. Liu C, Sun X and Chen J 2008 Application of human-simulating intelligent control in heating the super-high pressure kettle World Congress on Intelligent Control and Automation pp 1-4. 